\title{
A Rare Complication of Spinal Cord Ischemia Following Endovascular Aneurysm Repair of an Infrarenal Abdominal Aortic Aneurysm with Arteriosclerosis Obliterans: Report of a Case
}

\author{
Koichi Morisaki, MD, PhD, Takuya Matsumoto, MD, PhD, Yutaka Matsubara, MD, \\ Kentaro Inoue, MD, Yukihiko Aoyagi, MD, Daisuke Matsuda, MD, Shinichi Tanaka, MD, \\ Jun Okadome, MD, and Yoshihiko Maehara, MD, PhD
}

We herein report a case of a rare complication of spinal cord ischemia $(\mathrm{SCl})$ following endovascular aneurysm repair (EVAR). Computed tomography showed stenosis and calcification of bilateral iliac arteries and a saccular aneurysm of the terminal aorta. Paraplegia occurred soon after balloon angioplasty of iliac arteries and EVAR. Cerebrospinal fluid drainage was not performed because the patient was on dual antiplatelet drugs. The patient was treated with intravenous methylpredonisolone and naloxone; however, this did not improve his paraplegia. SCl after EVAR is extremely rare and unpredictable complication, however, physicians should be aware of $\mathrm{SCl}$ after EVAR in patients with atherosclerosis.

Keywords: spinal cord ischemia, endovascular therapy, abdominal aortic aneurysm, peripheral arterial disease

\section{Introduction}

Spinal cord ischemia (SCI) after endovascular aneurysm repair (EVAR) is rare with an incidence of approximately $0.21 \% .^{1)}$ The causes of SCI are derived from occlusion of the greater radicular artery, prolonged aortic clamping, intraoperative hypotension, atherosclerosis, embolization and interference with the pelvic circulation. We herein

Department of Surgery and Science, Graduate School of Medical Sciences, Kyushu University, Higashi-Ku, Fukuoka, Japan

Received: June 21, 2016; Accepted: August 1, 2016 Corresponding author: Koichi Morisaki, MD, PhD. Department of Surgery and Science, Graduate School of Medical Sciences, Kyushu University, 3-1-1, Maidashi, Higashi-Ku, Fukuoka, Fukuoka 812-8582, Japan

Tel: +81-92-642-5466, Fax: +81-92-642-5482

E-mail: morisaki@surg2.med.kyushu-u.ac.jp report a rare case of immediate-onset paraplegia following elective EVAR.

\section{Case Report}

A 75-year-old man complaining of intermittent claudication was admitted at our hospital. The patient has a previous history of hypertension, diabetes mellitus and hemodialysis due to diabetic nephropathy. A drug-eluting coronary artery stent was placed 2 months ago and dual antiplatelet therapy (DAPT) with aspirin and clopidogrel has been performed. The right ankle brachial pressure index (ABPI) was 0.92, 0.66 in the left leg. Computed tomography (CT) showed stenosis and calcification of the bilateral iliac arteries and a saccular aneurysm of the terminal aorta (Fig. 1). The left internal iliac artery was occluded. Elective EVAR was selected due to iliac stenosis with abdominal aortic aneurysm (AAA), which was the Trans-Atlantic Inter-Society Consensus II class D disease.

Under general anesthesia, endovascular treatment of the bilateral iliac arteries and EVAR using the Powerlink system (Endologix Inc, Irvine, Calif) were performed (Fig. 2). Anesthesia was induced with fentanyl, propofol and vecuronium and maintained with sevoflurane. The patient's initial blood pressure was 140/70 $\mathrm{mmHg}$. During the operation, a mean blood pressure of $60 \mathrm{mmHg}$ was observed. The operative time was $4 \mathrm{~h}$ and $43 \mathrm{~min}$ and the amount of intraoperative bleeding was $370 \mathrm{ml}$. However, a transfusion was not performed because hemoglobin concentration was $9.8 \mathrm{~g} / \mathrm{dl}$. Postoperative ABPI of the left leg was improved to 0.94 .

After awakening from anesthesia, the patient noted having bilateral lower extremity paraplegia. The neurological examination showed full muscle power over the upper limbs but no motor function below the T1 level with preserved sensory function. The patient was not able to undergo cerebrospinal fluid (CSF) drainage because the patient was 

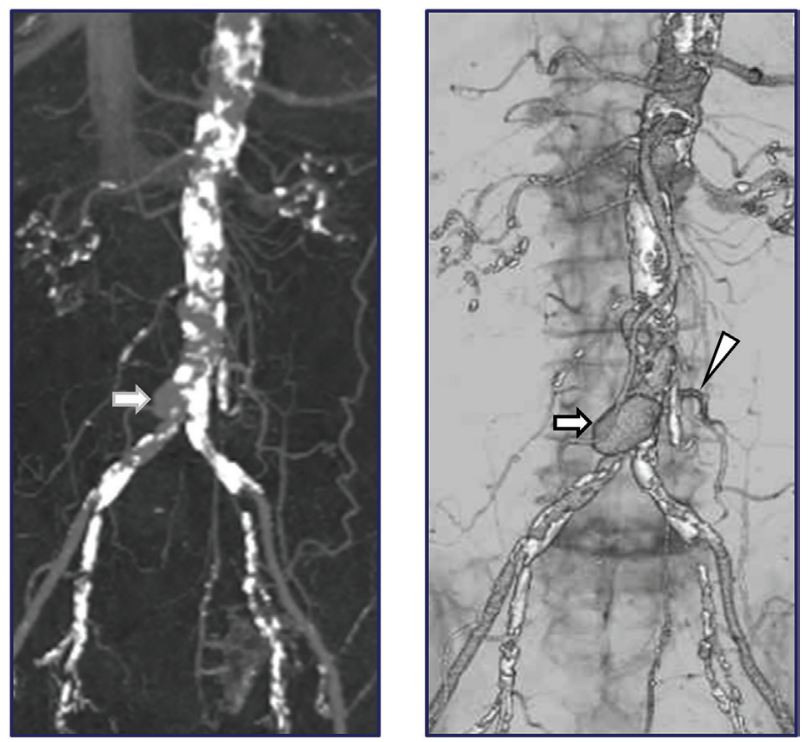

Fig. 1 Computed tomography showed stenosis of the bilateral iliac artery and a saccular aneurysm of the terminal aorta.
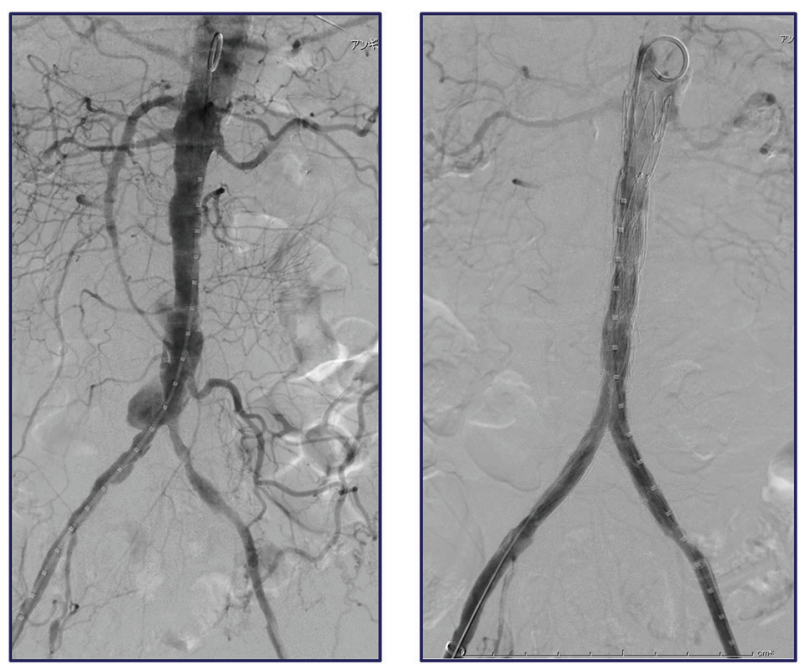

Fig. 2 Endovascular treatment to the bilateral iliac arteries and endovascular aneurysm repair using the Powerlink system were performed.

on DAPT. Intravenous methylprednisolone (1000 mg bolus and $200 \mathrm{mg} /$ day drip for $24 \mathrm{~h})$ and naloxone $(1 \mu \mathrm{g} / \mathrm{kg} / \mathrm{h}$ drip for 4 days) were administered. On the following day, magnetic resonance imaging (MRI) was performed, which showed high T2 signals in the spinal cord compatible myelopathy at the T12-L2 level (Fig. 3). Unfortunately, complete paraplegia remained and the patient was moved to another hospital in order to undergo rehabilitation.

\section{Discussion}

Paraplegia after EVAR is a rare complication, and an analysis of the Eurostar database of 2862 patients who

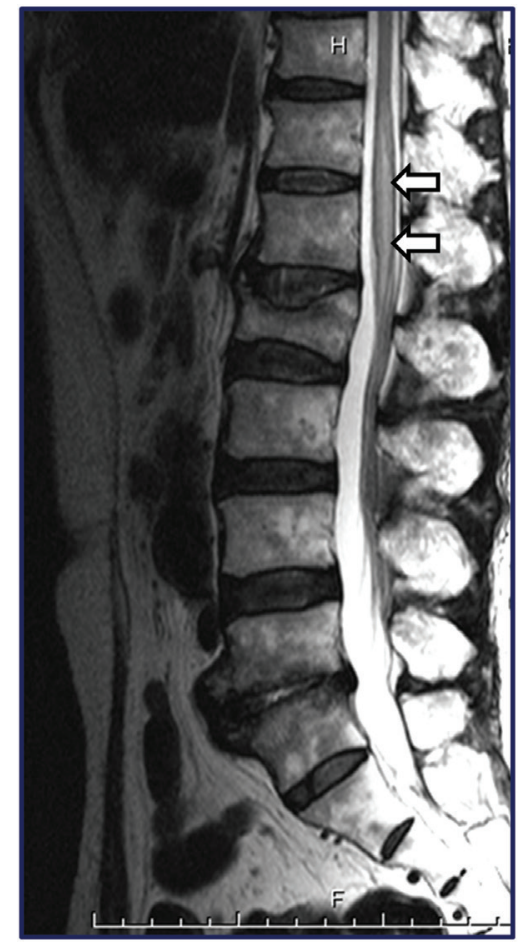

Fig. 3 Magnetic resonance imaging showed high T2 signals in the spinal cord as myelopathy at the T12-L2 level.

had undergone EVAR showed an incidence of $0.21 \%$ for SCI. ${ }^{1)}$ The cause of SCI after EVAR is not clearly understood. Various factors may contribute to SCI, such as occlusion of the greater radicular artery, prolonged aortic clamping, intraoperative hypotension, atherosclerosis, embolization and interference with the pelvic circulation. In patients who undergo EVAR, intraoperative hypotension, embolism and interruption of the collateral circulation from the iliolumbar and internal iliac arteries may be relevant.

The spinal cord is supplied by two posterior spinal arteries and one anterior spinal artery. Sensory tracts are supplied from posterior spinal arteries such as posterior and inferior cerebellar arteries, vertebral arteries and posterior radicular arteries. Conversely, motor tract is supplied from anterior spinal arteries such as the anterior radicular artery (Adamkiewicz). This artery originates from the T5-T7 intercostal arteries in $7 \%$. T8-T12 in $82 \%$, and from the lumbar artery at the level of L1-L2 in $11 \%$ of all cases. ${ }^{2)}$

In the present case, CT showed a thick iliolumbar artery, therefore, the spinal cord may be partially supplied by collateral circulation from iliolumbar artery. Initially, severe arterial sclerosis was detected and the left internal iliac artery was occluded; thus, the iliolumbar artery contributed to the collateral circulation.

Another possible cause of SCI in the present case is perioperative hypotension. A previous report showed 
that an intraoperative mean pressure of less than $70 \mathrm{mmHg}$ was a significant predictor of SCI. ${ }^{3)}$ In the present case, a mean pressure of less than $70 \mathrm{mmHg}$ was maintained for $2 \mathrm{~h}$, which may have caused SCI. Moreover, severe atherosclerotic change was observed in this patient, which may have worsened the circulation of the spinal cord.

In the present case, the procedure time was long because it was difficult to insert and remove the delivery system due to severe calcification. Final angiography showed no findings of dissection or rupture at iliac arteries as access site. Prolonged operative time and microembolism may be other contributing factors for SCI.

Regarding the treatment of SCI, CSF drainage, steroids, naloxone and maintenance of the blood pressure have been reported. CSF drainage is performed in order to decrease the cerebrospinal pressure thereby leading to an improvement of spinal cord perfusion. ${ }^{4)}$ Spinal cord perfusion pressure is the mean arterial blood pressure (MAP) minus the spinal fluid pressure; therefore, CSF drainage leads to the improvement of spinal cord perfusion. Steroids are used to suppress inflammation and reduce edema of the ischemic spinal cord. ${ }^{5)}$ Naloxone, an opioid receptor antagonist, reduces the risk of SCI through a potential mechanism of lowering excitatory amino acids such as glutamate in the CSF.6) However, the effectiveness and mechanism of naloxone therapy for SCI is unclear and naloxone is currently used according to previous experience. In the present case, CSF drainage was not performed because of the risk of bleeding due to DAPT. Therefore, systemic a steroid and naloxone were administered, however, the patient's paraplegia did not improve. Despite the patient has oral administration of antiplatelet therapy, CSF should have been performed in this case.

The cause of SCI after EVAR is unclear because of extremely rare complication. The risk factors for SCI in the treatment of a thoracic aortic aneurysm are as follows: endograft coverage of T9 to T12; over $20 \mathrm{~cm}$ of coverage of the thoracic aorta; occlusion of the left subclavian artery; $;^{7)}$ prior repair of an infrarenal AAA $;{ }^{4)}$ renal dysfunction; ${ }^{8)}$ and a low perioperative mean arterial pressure (MAP) of less than $70 \mathrm{mmHg} .{ }^{3)}$ It is difficult to predict the development of SCI preoperatively.

There was no occurrence of SCI after EVAR in approximately 300 case of EVAR in our institution. Almost case of EVAR had been performed by only incision of bilateral groins and there was no complication of bleeding. Therefore, antiplatelet therapy was continued, considering the risk of thrombotic event in coronary stent. In the present case, possible prevention for SCI was to maintain the MAP and preoperative cessation of antiplatelet drugs considering the necessity of CSF drainage. Furthermore, preoperative informed consent is crucial regardless of extremely rare complications in the treatment of AAA, as the occurrence of SCI could have serious adverse effects.

\section{Conclusion}

SCI after EVAR is extremely rare and unpredictable complication, however, physicians should be aware of SCI after EVAR in patients with atherosclerosis with atherosclerosis.

\section{Disclosure Statement}

The authors have no conflicts of interest to declare.

\section{Author Contributions}

Study conception: KM

Data collection: KM, TM

Analysis: KM, TM

Investigation: $\mathrm{KM}, \mathrm{TM}$

Writing: KM

Critical review and revision: all authors

Final approval of the article: all authors

Accountability for all aspects of the work: all authors

\section{References}

1) Berg P, Kaufmann D, van Marrewijk CJ, et al. Spinal cord ischaemia after stent-graft treatment for infra-renal abdominal aortic aneurysms. Analysis of the Eurostar database. Eur J Vasc Endovasc Surg 2001; 22: 342-7.

2) Koshino T, Murakami G, Morishita K, et al. Does the Adamkiewicz artery originate from the larger segmental arteries? J Thorac Cardiovasc Surg 1999; 117: 898-905.

3) Chiesa R, Melissano G, Marrocco-Trischitta MM, et al. Spinal cord ischemia after elective stent-graft repair of the thoracic aorta. J Vasc Surg 2005; 42: 11-7.

4) Schlösser FJ, Verhagen HJ, Lin PH, et al. TEVAR following prior abdominal aortic aneurysm surgery: increased risk of neurological deficit. J Vasc Surg 2009; 49: 308-14; discussion 314.

5) Laschinger JC, Cunningham JN, Cooper MM, et al. Prevention of ischemic spinal cord injury following aortic cross-clamping: use of corticosteroids. Ann Thorac Surg 1984; 38: 500-7.

6) Kunihara T, Matsuzaki K, Shiiya N, et al. Naloxone lowers cerebrospinal fluid levels of excitatory amino acids after thoracoabdominal aortic surgery. J Vasc Surg 2004; 40: 681-90.

7) Rizvi AZ, Sullivan TM. Incidence, prevention, and management in spinal cord protection during TEVAR. J Vasc Surg 2010; 52: 86S-90S.

8) Ullery BW, Cheung AT, Fairman RM, et al. Risk factors, outcomes, and clinical manifestations of spinal cord ischemia following thoracic endovascular aortic repair. J Vasc Surg 2011; 54: 677-84. 